und 1955 die Royal-Medaille; mehrere Hochschulen haben ihm die Würde eines Ehrendoktors verliehen: Der Beginn der Arbeiten auf dem Nukleotid-Gebiet liegt etwa 15 .Jahre zurück: die erste Mitteilung zum Problem der Synthese von Purin-Nukleosiden findet sich im Journal of the Chemical Society des Jahres 1943. Rasch wurde es nötig, neue Methoden zu entwickeln. 1945 erschien die erste Arbeit über Phosphorylierungs-Studien. In dasselbe Jahr fällt der Beweis für die Richtigkeit der Lohmannschen Adenosin-triphosphat-Formel. Einer ersten Mitteilung über synthetische Arbeiten auf dem Nukleotid-Gebiet (1947) folgte bald ein erster Bericht über die Synthese des Adenosintriphosphats (1948/49). 1949 waren die Studien uber die Konstitution der Nukleoside abgeschlosseri; und in das Jahr 1952 fällt der Beginn von Untersuchungen iiber die Struktur und chemischen Eigenschaften der Nukleinsäuren. Gleichzeitig wandte ToDD am Beispiel des Elavin-adenin-dinukleotids seine Aufmerksamkeit erstmals der Synthese der Nukleotid-Coenzyme zu. Der erfolgreichen Synthese der genannten Verbindung folgte die Synthese von Uridin-diphosphat-glukose (1954/56), Uridin-diphosphatgalaktose (1956) und Diphospho-pyridin-nukleotid (1957). Andere Arbeiten, welche ToDD bekannt gemacht haben, sind seine Untersuchungen iber Haschisch ${ }^{1}$ und die Ermittlung der Konstitution von Vitamin $\mathrm{B}_{12}$ (1955).

Professor TODD ist ein hervorragender Organiker, der unter dem Eindruck einer guten biochemischen Schulung sein Können konsequent in den Dienst der Biochemie gestellt hat. Unter dem Titel «The Impact of Chemistry on Biology and Medicine" schreibt er in einem Aufsatz: "The chemist's position is very neatly put in a remark of the late Sir FREDERICK Hopkins who said of him 'His may not be the last word in the description of life, but without his help the last word will never be said'y. Sir Alexinder TODd hat diesen Worten nachgelebt. Der Beitrag, welcher von ihm zur Beschreibung des Lebens geleistet wurde, hat mit der Erteilung des Nobelpreises eine Anerkennung gefunden, der jedermann mit Überzeugung zustimmen wird.

M. BRENNER

\section{Daniel Bovet}

Le prix Nobel de physiologie et de médecine vient d'être attribué à M. DANIEL BOveT, chef du Laboratoire de chimie thérapeutique de l'Istituto superiore di sanità, a Rome. Il couronne une ouvre scientifique aussi importante par ses aspects théoriques et par les voies nouvelles qu'elle a ouvertes que par ses applications médicales.

Né à Neuchâtel (Suisse) en 1907, M. Bover a fait ses études à la faculté des sciences de l'Université de Genève. A 22 ans, il entre à l'Institut Pasteur de Paris, dans le laboratoire de Fourneau. Il y rencontre Mle NitT qui deviendra sa femme et une admirable collaboratrice. C'est en 1947 que les Bovet s'établissent à Rome.

A l'Institut Pasteur, M. Bover participe d'abord à des recherches dans le domaine de la chimiothérapie. Avec M. ef Madame TrÉFoü̈L et Federicio NitTI, il démontre en 1935 que le prontosil dont Domagk venait de découvrir les proprietés anti-infectieuses subit dans les tissus une scission de sa liaison azoïque et fournit la para-aminobenzène-sulfonamide. C'est cette substance qui constitue la fraction active de la molécule du prontosil et c'est d'elle que dérivent les nombreux sulfamidés dont la médecine dispose actuellement.

C'est à la pharmacologie chimique du système nerveux végétatif que $M$. Bover, par la suite, consacre son

1 A. R. TodD, Exper. 2, 55 (1946).

2 Reviews of pure and applied Chemistry 1, 14 (1951). activité. Dominant avec une égale maitrise les problèmes de la synthèse chimique et ceux de la pharmacodynamie, il effectue ses recherches les plus importantes dans le domaine des sympatholytiques, des anti-histaminiques et des poisons curarisants.

Au cours de ses recherches sur les sympatholytiques, substances bloquant les effets du système nerveux sympathique et de ses médiateurs adrénergiques, M. BoVET rapporte dès 1933 les effets puissants de certains benzodioxanes, le $883 \mathrm{~F}$ (Prosympal) et le $933 \mathrm{~F}$ (Piperoxan). Cette dernière substance rend de précieux services dans le diagnostic des, tumeurs fonctionnelles de la médullo-surrénale.

En 1937, soit plus d'un quart de siècle après que DALE eût étudié la pharmacologie de l'histamine et découvert son rôle dans le choc anaphylactique, M. Bovet et ses collaborateurs démontrent pour la première fois les propriétés anti-histaminiques de certains éthers phénoliques dont le plus actif, le $929 \mathrm{~F}$, protège le cobaye contre le choc histaminique. De nombreux antagonistes de l'histamine furent ensuite étudiés par M. Bovet, en particulier un dérivé de l'aminopyrine, le néoantergan, l'un des. plus puissants anti-histaminiques connus. L'utilité clinique de tels produits dans le traitement de phénomènes allergiques se révéla si grande que des milliers d'anti-histaminiques ont été synthétisés depuis lors dans de nombreux laboratoires.

Poursuivant leurs recherches sur la pharmacologie des transmissions neuro-effectrices, M. Bover et ses collaborateurs ont fait, ces dernières années, de remarquables découvertes dans le domaine des curarisants de'synthèse. C'est en effet en 1946 qu'ils réussissent la première synthèse d'une substance curarisante, réalisêe sur le modèle de la $d$-tubocurarine et dont la sélectivité pour la jonction neuromusculaire est comparable à celle de cet alcaloïde naturel du curare. Une série de synthèses de structures de plus en plus simplifiées, aboutissant à la découverte du Flaxédil, permettent de préciser les caractéristiques chimiques des produits curarisants et les rapports entre leur structure et celle de l'acétylcholine, le médiateur de la transmission neuromusculaire. L'action curarisante de la succinyIcholine dont la molécule est le "doublet" de celle de l'acétylcholine est également découvèrte. Flaxédil et succinylcholine ont pris place parmi les adjuvants les plus précieux de l'anesthésie chirurgicale car ils permettent d'obtenir une relaxation musculaire sans provoquer divers effets indésirables des alcaloïdes du curare; de plus, la-durée de leur action est aisément réglable ${ }^{I}$

L'œuvre de M. Bovet, qui a rendu des services inestimables à la médecine dans les domainés les plus variés, revêt également une importance théorique considérable par les lumières qu'elle apporte sur les relations entre la structure chimique des composés et leurs effets. Dans la préface de leur ouvrage: Structure et activité pharmacodynamique des médicaments du systeme nevveux végétatif, M. Bovit et Mme Bovei-Nitis écrivent: "Les rapports entre la constitution chimique des corps et leur action pharmacodynamique sera, à notre sens, l'aboutissemment et la justification de toute recherche pharmacodynamique," On ne saurait mieux définir la ligne directrice de l'œuvre de M. Daniel Bovet.

\section{Gorrigendum}

J. Posternak

A. FRey-Wyssling und S. Bäbler: Zur Biochemie des Gezuächshaustabaks. Experientia Vol. XIII, Heft 10, S. 399 (1957). Auf Seite 399, rechte Kolonne, gehört der zweite Abschnitt « Es scheint also ... zu stimulieren vermag unter die Tabelle.

1 D. Bovet-Nitri, Exper. 4, 325 (1948). 